showed that 128 children and 199 young adults suffered from AIS. Children were more likely to be male than young adults $(62 \% / 49 \%, \mathrm{p}=0.023)$. Children were less frequently hypertensive, and less likely to have hypercholesterolemia and family history of stroke. Stroke severity was similar in children and young adults. The etiology of stroke in children was more likely to be "other determined cause." Cervicocerebral artery dissections were less frequent in children. Outcome was similar, and favorable in 59\% children and $60 \%$ of young adults. Mortality was also similar, $4 \%$ and $6 \%$. A low score on the pediatric version of the National Institutes of Health stroke scale was the most important predictor of favorable outcome $(\mathrm{p}<0.001)$. (Bigi S, Fischer U, Wehrli E, et al. Acute ischemic stroke in children versus young adults. Ann Neurol August 2011;70:245-254). (Respond: Prof Dr Steinlin, Division of Neuropediatrics, University of Bern, Inselspital, Freiburgstrasse 4, 3010 Bern, Switzerland. E-mail: maja.steinlin@insel.ch).

COMMENT. Stroke severity and clinical outcome at 3 to 6 months are similar in children and young adults but stroke etiology and risk factors are different.

\title{
SEIZURES AS PRESENTING SYMPTOM OF ARTERIAL STROKE
}

Researchers at The Children's Hospital of Philadelphia, PA, determined the incidence of seizures as a presenting symptom of acute arterial ischemic stroke (AIS) in children and identified risk factors for seizures. Among 60 children aged 2 months to 18 years with AIS, 13 presented with seizures (22\%), focal in 10 . Of 47 children not presenting with seizures, only 2 had clinical seizures later in the hospitalization period. Seizure was the heralding sign of stroke onset in children presenting with seizures, preceding other focal neurologic signs and symptoms. Younger age was a risk factor for seizures at presentation ( 1.1 years with seizures vs 10 years with no seizure; $\mathrm{p}=0.0009$ ). Occurrence of seizures was not associated with infarct location or etiology. All patients with seizures had hemiparesis. Three of 4 children with clinical seizures also had nonconvulsive seizures on continuous EEG monitoring at presentation. (Abend NS, Beslow LA, Smith SE, et al. Seizures as a presenting symptom of acute arterial ischemic stroke in childhood. J Pediatr September 2011;159:479-483). (Reprints: Nicholas S Abend MD, Division of Neurology, Children's Hospital of Philadelphia, 3501 Civic Center Blvd, Philadelphia, PA 19104. E-mail: abend@email.chop.edu).

COMMENT. Stroke should be considered in a child presenting with new-onset seizure in combination with focal neurologic deficits. Seizures are rare during acute hospitalization for AIS if they do not occur at presentation, and prophylactic anticonvulsant therapy is probably not warranted. Nonconvulsive seizures, detected by continuous EEG monitoring, may be an under-recognized complication of AIS, and EEG should be considered in patients with impaired consciousness. 